\title{
LETTER
}

\section{Number needed to treat and cost-effectiveness in the prevention of ventilator-associated pneumonia}

\author{
Duncan Wyncoll* and Luigi Camporota
}

While clinicians continue to redefine ventilator-associated pneumonia (VAP), numerous innovations that claim to reduce pulmonary microaspiration and its consequences - that is, novel endotracheal cuff shapes and cuff materials, subglottic drainage, automatic cuff pressure controllers, oral anti-septics, selective digestive decontamination (SDD), and devices to combat biofilm formation within the lumen of the tracheal tube - are coming to the market $[1,2]$. There are two questions that clinicians ask when deciding whether to incorporate a new product or intervention into a VAP prevention bundle. Firstly, what are its efficacy and effectiveness? In other words, what is the relative risk reduction (RRR) and therefore the number needed to treat (NNT) to prevent one additional VAP. Secondly, is this new intervention cost-effective in my local patients?

To answer the first question, one needs data from clinical trials and the knowledge of the baseline VAP rate with the likely RRR of the local case mix. We have calculated (Table 1) the NNT required to prevent one additional VAP for patients who require intubation and mechanical ventilation (MV) for more than 72 hours and an average time of MV of 10 days. The NNTs are based on an RRR ranging from $5 \%$ to $50 \%$ and a control event rate for VAP ranging from $1 \%$ to $20 \%$, given a uniform distribution of NNTs across the range of RRRs. For example, with a VAP rate of approximately $8 \%$ and an intervention that reduces VAP by $45 \%$, the NNT is $28-\mathrm{a}$ scenario that is realistic given a recent meta-analysis of one particular intervention [3].

To establish whether the intervention is cost-effective, further knowledge of the cost of the intervention and the cost to treat an episode of VAP is required. A recent US study estimated the cost of VAP to be nearly $\$ 40,000$ $(£ 25,000$ or $€ 30,000)$ [4]. If costs are assumed to be lower in Europe, then a conservative estimate of the cost per episode of VAP would still be around $£ 10,000$, which is

*Correspondence: duncan.wyncoll@gstt.nhs.uk

Department of Adult Critical Care, Guy's and St Thomas' NHS Foundation Trust, St Thomas' Hospital, 1st Floor East Wing, Lambeth Palace Road, London, SE1 7EH, UK equivalent to an extra 7 days of intensive care unit (ICU) stay. What should we consider when assessing the costeffectiveness of VAP prevention?

We have calculated (Table 2) the additional money (in pounds) that can be spent to prevent an episode of VAP (per 10 days of MV) to achieve cost-neutrality. If we assume a hypothetical VAP cost of $£ 10,000$, then with a VAP rate of $8 \%$ and an RRR of $45 \%$, it is cost-effective to spend up to $£ 360$. Furthermore, even for an ICU with a VAP rate of only $4 \%$ and an intervention that reduces VAP by just $25 \%$, it is still cost-effective to spend up to $£ 100$ per 10 days of MV. It should be noted that some VAP prevention interventions (for example, a modified tracheal tube cuff) require just a 'one-off' initial cost whereas other interventions (for example, SDD) require an 'ongoing' daily cost.

We think that this analysis might help clinicians in making the important economic decision of whether to adopt a new VAP prevention device or procedure. Our calculations can easily be adapted to local currencies and circumstances worldwide.

\section{Abbreviations}

ICU, intensive care unit; MV, mechanical ventilation; NNT, number needed to treat; RRR, relative risk reduction; SDD, selective digestive decontamination; VAP, ventilator-associated pneumonia.

\section{Competing interests}

LC declares that he has no competing interests. DW has given paid lectures or consulted for Kimberly-Clark (Irving, TX, USA), Covidien (Mansfield, MA, USA), ConvaTec (Skillman, NJ, USA), Iskus Health (Dublin, Ireland), Sage Products (Cary, IL, USA), Eli Lilly and Company (Indianapolis, IN, USA), and Pfizer Inc (New York, NY, USA) and has a stock interest in Biovo Technologies (Tel Aviv, Israel). The authors declare that they have no personal financial interests.

\section{Published: 7 June 2012}

\section{References}

1. Zolfaghari PS, Wyncoll DL: The tracheal tube: gateway to ventilatorassociated pneumonia. Crit Care 2011, 15:310.

2. Coppadoro A, Bittner E, Berra L: Novel preventive strategies for ventilatorassociated pneumonia. Crit Care 2012, 16:210.

3. Muscedere J, Rewa O, McKechnie K, Jiang X, Laporta D, Heyland DK: Subglottic secretion drainage for the prevention of ventilator-associated pneumonia: a systematic review and meta-analysis. Crit Care Med 2011, 39:1985-1991.

4. Kollef MH, Hamilton CW, Ernst FR: Economic impact of ventilator-associated pneumonia in a large matched cohort. Infect Control Hosp Epidemiol 2012, 33:250-256. 
Table 1. Number needed to treat in ventilator-associated pneumonia

\begin{tabular}{|c|c|c|c|c|c|c|c|c|c|c|}
\hline & \multicolumn{10}{|c|}{ Relative risk reduction } \\
\hline & $5 \%$ & $10 \%$ & $15 \%$ & $20 \%$ & $25 \%$ & $30 \%$ & $35 \%$ & $40 \%$ & $45 \%$ & $50 \%$ \\
\hline \multicolumn{11}{|c|}{ Baseline VAP rate } \\
\hline $1 \%$ & 2,000 & 1,000 & 667 & 500 & 400 & 333 & 286 & 250 & 222 & 200 \\
\hline $2 \%$ & 1,000 & 500 & 333 & 250 & 200 & 167 & 143 & 125 & 111 & 100 \\
\hline $4 \%$ & 500 & 250 & 167 & 125 & 100 & 83 & 71 & 63 & 56 & 50 \\
\hline $6 \%$ & 333 & 167 & 111 & 83 & 67 & 56 & 48 & 42 & 37 & 33 \\
\hline $8 \%$ & 250 & 125 & 83 & 63 & 50 & 42 & 36 & 31 & 28 & 25 \\
\hline $10 \%$ & 200 & 100 & 67 & 50 & 40 & 33 & 29 & 25 & 22 & 20 \\
\hline $15 \%$ & 133 & 67 & 44 & 33 & 27 & 22 & 19 & 16.7 & 15 & 13 \\
\hline $20 \%$ & 100 & 50 & 33 & 25 & 20 & 17 & 14 & 12.5 & 11 & 10 \\
\hline
\end{tabular}

Number needed to treat (NNT) was calculated as: NNT [relative risk of event] $=1$ / (pc $\times$ RRR), where pc is the proportion of control group subjects who suffer an event and RRR is relative risk reduction. These NNTs are based on events per 10 days of mechanical ventilation, meaning that more than one event can occur in a single patient who is ventilated for more than 10 days. VAP, ventilator-associated pneumonia.

Table 2. Cost-effectiveness of an intervention based on baseline ventilator-associated pneumonia rate and its relative risk reduction

\begin{tabular}{|c|c|c|c|c|c|c|c|c|c|c|}
\hline & \multicolumn{10}{|c|}{ Relative risk reduction } \\
\hline & $5 \%$ & $10 \%$ & $15 \%$ & $20 \%$ & $25 \%$ & $30 \%$ & $35 \%$ & $40 \%$ & $45 \%$ & $50 \%$ \\
\hline \multicolumn{11}{|c|}{ Baseline VAP rate } \\
\hline $1 \%$ & $£ 5$ & $£ 10$ & $£ 15$ & $£ 20$ & $£ 25$ & $£ 30$ & $£ 35$ & $£ 40$ & $£ 45$ & $£ 50$ \\
\hline $2 \%$ & $£ 10$ & $£ 20$ & $£ 30$ & $£ 40$ & $£ 50$ & $£ 60$ & $£ 70$ & $£ 80$ & $£ 90$ & $£ 100$ \\
\hline $4 \%$ & $£ 20$ & $£ 40$ & $£ 60$ & $£ 80$ & $£ 100$ & $£ 120$ & $£ 140$ & $£ 160$ & $£ 180$ & $£ 200$ \\
\hline $6 \%$ & $£ 30$ & $£ 60$ & $£ 90$ & $£ 120$ & $£ 150$ & $£ 180$ & $£ 210$ & $£ 240$ & $£ 270$ & $£ 300$ \\
\hline $8 \%$ & $£ 40$ & $£ 80$ & $£ 120$ & $£ 160$ & $£ 200$ & $£ 240$ & $£ 280$ & $£ 320$ & $£ 360$ & $£ 400$ \\
\hline $10 \%$ & $£ 50$ & $£ 100$ & $£ 150$ & $£ 200$ & $£ 250$ & $£ 300$ & $£ 350$ & $£ 400$ & $£ 450$ & $£ 500$ \\
\hline $15 \%$ & $£ 75$ & $£ 150$ & $£ 225$ & $£ 300$ & $£ 375$ & $£ 450$ & $£ 525$ & $£ 600$ & $£ 675$ & $£ 750$ \\
\hline $20 \%$ & $£ 100$ & $£ 200$ & $£ 300$ & $£ 400$ & $£ 500$ & $£ 600$ & $£ 700$ & $£ 800$ & $£ 900$ & $£ 1,000$ \\
\hline
\end{tabular}

Values $(£)$ refer to the average additional expense that can be spent for an intervention, per 10 days of mechanical ventilation, for it to be cost-neutral assuming a ventilator-associated pneumonia (VAP) cost of $£ 10,000$

doi:10.1186/cc11346

Cite this article as: Wyncoll D, Camporota L: Number needed to treat and cost-effectiveness in the prevention of ventilator-associated pneumonia. Critical Care 2012, 16:430. 